\title{
LAS INDUSTRIAS ACHELENSES DE ANDALUCÍA: ORDENACIÓN Y COMENTARIOS
}

\section{ACHEULIAN INDUSTRIES IN ANDALUSIA: CHARACTERISATION AND DISCUSSION}

\author{
por \\ ENRIQUE VALLESPI PEREZ
}

\begin{abstract}
A la memoria del amigo José Ramos Cabello: homenaje a la proyección social de su vida personal y docente y recuerdo del cursillo, sobre este estudio, en la Universidad en La Axarquía

(Vélez-Málaga, agosto de 1989)
\end{abstract}

RESUMEN En la periferia meridional de la Península Ibérica que integra el territorio andaluz se prefigura, sobre bases cronoestratigráficas de la Depresión de Guadix-Baza y aledaños y de las terrazas fluviales de la Depresión Inferior del Guadalquivir, una secuencia regional estándar del Paleolítico Inferior, con indicios y evidencias iniciales del Pleistoceno inferior avanzado y del Pleistoceno medio antiguo, y una seriación de industrias achelenses del Pleistoceno medio pleno y reciente y de su perduración inmediata trabada a las secuencias de un Paleolítico Medio diferenciado en postachelenses de ámbitos fluviales y musterienses clásicos de cuevas. Historiografía y bibliografía, yacimientos y localizaciones y planteamiento del estudio, en una primera visión regional de conjunto.

ABSTRACT In the southern periphery of the Iberian Peninsula which makes up the Andalusian territory, an outline is given of a standard regional sequence of the Early Palaeolithic on chronostratigraphic bases of the Depression of Guadix-Baza and its outskirts and of the Lower Depression of the Guadalquivir. This sequence contains initial vestiges and evidence of the advanced Early Pleistocene and a serie of Acheulian industries at the height of the Plein Middle and Late Pleistocene and of its immediate endurance which is linked to the sequence of a Middle Palaeolithic, which is characterized as postacheulian in a fluvial context and as clasical mousterian from caves. Historiography and bibliography, deposits and locations and study plannig, in a preliminary but complete regional picture. 
En consonancia con el panorama general del Paleolítico Inferior de la Península Ibérica, en la periferia meridional que integra el territorio andaluz los indicios y evidencias iniciales de atribución mantenida hasta el momento al Pleistoceno Inferior avanzado y transicional permanecen aún sin aclarar, mientras que empiezan a vertebrarse las industrias achelenses del Pleistoceno Medio Pleno y Reciente. En efecto, mantenido el impasse de Orce, en las importantes perspectivas de la cuenca de Guadix-Baza, y apartado El Aculadero de una atribución tan antigua, y sin verificarse alguna otra atribución pendiente, la documentación inferopaleolítica actual de la región corresponde toda ella al Pleistoceno Medio y su prolongación transicional y está constituída por el indicio del Paleolítico Inferior Antiguo indeterminando de Cúllar de Baza I, los conjuntos achelenses del interior de los depósitos de las terrazas fluviales de la Depresión Inferior del Guadalquivir y el horizonte achelense avanzado y de perduración de las terrazas bajas de dicho ámbito fluvial y de los yacimientos de la Solana del Zamborino y la base estratigráfica de Cueva Horá, además de las consiguientes series achelenses y postachelenses de superficie asimilables. La comprobación de la existencia de industrias achelenses en los depósitos secuenciales de las terrazas fluviales de la Depresión Inferior del Guadalquivir es un hecho novedoso que viene a cubrir el vacío del pleno Pleistoceno Medio, existente hasta ahora en la región, entre los citados yacimientos conocidos de Cúllar de Baza I y de la Solana del Zamborino y Cueva Horá, lo que supone la prefiguración de una secuencia achelense regional, a cuyo planteamiento, de indudable interés en sus perspectivas generales, pretende contribuir, como primera visión de conjunto, la presente entrega.

\section{HISTORIOGRAFIA}

La introducción que antecede refleja la situación actual, de planteamiento de una secuencia regional estándard del Paleolítico Inferior en Andalucía, compromiso de los investigadores del momento presente y resultado a su vez de una historia de recogida de datos sobre la que conviene reflexionar, porque puede ayudar a esclarecer nuestras perspectivas del estudio.

Aparte del precedente de alguna atribución más o menos voluntarista, la recogida de datos para la elaboración de un conocimiento científico del Paleolítico Inferior de Andalucía (en su sentido actual, de separación del Musteriense como Paleolítico Medio) comienza en la segunda década del presente siglo, con el descubrimiento y publicación de los dos primeros yacimientos registrados, La Janda y Puente Mocho, en intervención de los modeladores del Paleolítico ibérico, Breuil (1914 y 1917) y Obermaier (1916 y 1925) y de los grandes pioneros Hernández Pacheco (1915) y Cabré y Wernert (1916). Conviene anotar la corrección relativa de los criterios metodológicos aplicados en estas primeras localizaciones de nuestras industrias en superficie: la preocupación sobre la posición de las industrias en relación con las formaciones cuaternarias sustentantes, a lo que Breuil dedicó su trabajo de 1917 sobre La Janda; y en lo tipológico, la separación de las muestras en series por grados de rodamiento y la observación de la existencia de piezas con concreciones de matriz, efectuadas por Paul Wernert en su análisis de las muestras de Puente Mocho, en su aludido trabajo con Cabré, de 1916 (p. $17 \mathrm{y}$, para los criterios de rodamiento, p. 17-18,20 y 21).

Ambos yacimientos, junto a otros hallazgos andaluces menores, pasaron al corpus de El Hombre Fósil de Obermaier, incorporándose con ello a la bibliografía general (Obermaier, 1916 y 1925).

Desde entonces, con la publicación en 1925 de la segunda edición de dicha obra, quedó truncada la proyección peninsular que auguraban las perspectivas de tales hallazgos andaluces, sin otra actividad regional reseñable que la actuación local en Córdoba en 1931 del geólogo A. Carbonell, valorable, por 
cierto, como primera intervención no foránea, a tener en cuenta. De este modo, los descubrimientos iniciales quedaron en un mero episodio activo aislado, sin continuidad en la región hasta transcurrida la mitad del siglo.

La tardía reanudación de las tareas se limitó, en un primer momento, en los años 50 y 60 , a la comprobación de la existencia de industrias inferopaleolíticas de cantos tallados, con la sospecha de su vinculación estratigráfica a las terrazas del Guadalquivir, en Carmona (por Martínez Santa-Olalla, con una comunicación de Sáez Martín, 1956) y a sendas llamadas de atención a las posibilidades del Paleolítico Inferior regional, en relación con las terrazas fluviales, explicadas por la teoría climática (De Terra, 1956) y con la riqueza paleontológica de sus formaciones pleistocénicas (Aguirre, Collins, Cuenca, 1962). Tales aportaciones y estímulos no tuvieron, lamentablemente, repercusión inmediata, puesto que la actividad autóctona era en esos años mínima, reducida a alguna localización en la provincia de Córdoba (Casas Morales, 1964).

Poco después, al final de la década y en los comienzos de los años 70, se anunciaban las localizaciones de industrias de cantos tallados efectuadas por el geólogo C. Viguier en la Depresión Inferior del Guadalquivir, en superficie, en las terrazas del Guadlaquivir en Carmona y la costa de El Rompido, y su conexión estratigráfica en El Aculadero, del litoral de Cádiz; avalados por Bordes y con la participación directa de Thibault (Bordes y Viguier, 1969 y 1971; Viguier y Thibault, 1973; Viguier, 1974), estos descubrimientos (cuyos autores, por cierto, ignoraron en sus publicaciones el precedente, trece años anterior, de Martínez Santa-Olalla) cobrarían proyección inmediata.

Entretanto se habían iniciado ya las primeras tareas de excavación en yacimientos ínferopaleolíticos andaluces: en 1972 en La Solana del Zamborino y al año siguiente en Cúllar de. Baza I, por la Universidad de Granada, con Botella de Ruíz Bustos, y se iniciaron también, en 1973, en el Aculadero, por las Universidades de Burdeos y Complutense de Madrid, con Thibault, Querol, Viguier y Santonja.

Desde entonces se asite a una generalización de las tareas, atendidas en adelante desde la propia región, con una continua recogida de datos, de localizaciones y registros de evidencias, cuyas entregas se publicaron de manera continuada, cubriendo prácticamente sin interrupción el transcurso de los años hasta el momento actual, en actuaciones individualizadas desde 1975 hasta la institucionalización autonómica de las tareas a partir de 1985.

En ese decenio de generalización de las actividades destaca en primer lugar la consecución de las excavaciones emprendidas, con publicación de los resultados: yacimiento y datación de Cúllar de Baza I (Ruiz Bustos, 1976; Ruiz Bustos y Michaux, 1976; Ruiz Bustos 1984); yacimiento e industria de La Solana del Zamborino (Botella y otros, 1976) y la memoría de El Aculadero (Querol y Santonja, 1983). Simultáneamente se sucedían las publicaciones del despliegue de prospecciones diversas sobre localizaciones de superficie, con aportaciones de desigual valor, en el alto Guadalquivir, de la provincia de Jaén (Sánchez Martínez, 1975; López Páyer y Soria Lerma, 1976; Sánchez Martínez y García García, 1977; y Carrasco y otros, 1979; Carrasco, Toro y otros, 1981) y más esporádicas en las provincias de Málaga (Leiva, 1977; Solanes y García León, 1983), Huelva (Del Amo, 1976) y Cádiz (Giles y Sáez, 1980; Carbonell y Canal, 1981), y reducidas en la provincia de Córdoba a dos aportaciones de 1976, inéditas entonces y publicada con posterioridad una de ellas (Santonja y Querol, 1988; y Beriguistáin, inédita).

Más alcance tendría, por su continuidad y resultados, el planteamiento de la prospección sistemática de la Depresión Inferior del Guadalquivir, emprendida por el Departamento de la Universidad de Sevilla, desde 1978 por E. Vallespí y G. Alvarez, con aportaciones de localizaciones de superficie de la provincia de Huelva (Vallespí y otros, 1981) y principalmente la comprobación de una secuencia del Paleolítico Inferior en las terrazas del Bajo Guadalquivir de la provincia de Sevilla, con industrias en 
conexión con los depósitos detríticos y abundantes localizaciones de superficie (Vallespí, Alvarez, Amores y Escacena, 1982; Amores, 1982; y Vallespí, 1983).

Al final de la etapa tendría lugar el descubrimiento del fragmento craneal y su atribución humana del yacimiento paleontológico de Venta Micena, en Orce (Gibert, Agustí y Moyá, 1983), cuya difusión desbordaría los cauces netamente científicos, por una serie de razones (puestas en consideración por Vega Toscano, 1984; y Vallespí, 1988 y e.p.).

Sociológicamente el despliegue de las tareas de este último decenio supone, junto al necesario mantenimiento de intervenciones desde fuera, la particiapción creciente por parte de los estudiosos e investigadores desde la propia región, aunque siguiera faltando la activa responsabilización institucionalizada de los centros andaluces específicamente implicados, como los Departamentos de Prehistoria y Arqueología de sus Universidades, que mostrarían sus carencias docentes e investigadoras en este campo ínferopaleolítico concreto de trabajo, evidenciadas de modo tácito en su falta de atención corporativa y explícitamente en algún intento desafortunado de trabajo académico sobre determinadas series de industrias de superficie.

En esta situación, asumió la Junta de Andalucía las competencias autonómicas de Cultura, creándose en Octubre de 1984 la Comisión Andaluza de Arqueología, órgano consultivo de la Consejería de Cultura, asesor de la Dirección General de Bellas Artes (después, de Bienes Culturales), lo que supondrá la paulatina institucionalización de las tareas arqueológicas en el ámbito de la Comunidad (cfs. la reseña de la efeméride en Anuario Arqueológico de Andalucía,1985, I: 5-9).

Muy tempranamente, con anterioridad a esta asumpción de competencias, la propia Junta de Andalucía inició su actuación arqueológica autonómica con dos actos que afectan al estudio del Paleolítico Inferior: un Primer Encuentro de Prehistoria Andaluza, celebrado en Málaga en Junio de 1982, con ponencia sobre el Estado actual del conocimiento de la Prehistoria Andaluza, a cargo de P. Acosta y E. Vallespí, cuya parte de mi responsabilidad constituyó una primera ordenación conjunta del Paleolítico y Epipaleolítico regionales, y con informes de la situación provincial de los estudios, debido a los directores de los respectivos museos, cuyas aportaciones han quedado inéditas, entregadas para unas Actas que no han llegado a publicarse; y el otro acto en cuestión, el anulado Coloquio sobre el Hombre de Orce, proyectado para su celebración en mayo de 1984, en Granada, y cuyo aplazamiento a última hora lo dejó sin efecto; su programa, del que fui responsable como coordinador científico, establecía cuatro sesiones de trabajo, con la temática e intervenciones siguientes: I, Presentación del hallazgo por el equipo de excavación, J. Agustí, S. Moyá-Solá, J. Gibert e I. Toro; II, Comunicaciones de Geología y Paleontología: J. Chaline y G. Brochet; M. Hoyos, J. Peña, A. Ruiz Bustos y C. Dabrío; A. Ruiz Bustos y T. Alberdi; y J. F. de Villalta; III, Comunicaciones de Arqueología: F. Gusi; E. Vallespí; E. Carbonell y R. Mora; L. Raposo; M. Botella; y M. A. Querol y M. Santonja; IV, Síntesis E. Bonifay; M. A. de Lumley; H. de Lumley; y E. Aguirre. Algunos pormenores de la anulación del Coloquio han quedado expuestos en otro lugar (Vallespí, 1984).

La constitución de la Comisión de Arqueología coincidió con el Congreso Homenaje a Luis Siret; organizado asimismo por la Junta de Andalucía y celebrado en Cuevas de Almanzora en el mismo junio de 1984 y cuyas Actas publicadas en 1986 constituyen la puesta al día de la Prehistoria regional, de obligada referencia bibliográfica en las nuevas tareas de la etapa actual, que queda con todo ello virtualmente iniciada. De la institucionalización de las tareas desde entonces conviene destacar el encauzamiento de las tradicionales campañas de campo hacia proyectos de investigación programados y la implantación de las Jornadas de Arqueología Andaluza, en el mismo año 1985, junto a la creación de los Anuarios para recoger los informes de las campañas e intervenciones de urgencia anuales, habiendo aparecido los de 1985, 1986 y 1987. Con respecto al Paleolítico Inferior hay que destacar la 
dignificación de las tareas de campo, resultado de la positiva contemplación de las prospecciones superficiales sistemáticas en la nueva legislación autonómica, y un incremento de publicaciones, con elevación apreciable del nivel medio de sus contenidos.

En las Actas del Homenaje a Luis Siret, publicadas en 1986, pero referidos sus estudios a 1984, año de celebración de sus sesiones, se inician oportunamente las publicaciones de esta etapa reciente con la inclusión de una primera visión de conjunto del Paleolítico Inferior y Medio regionales, de planteamiento de las nuevas líneas generales de trabajo y recopilación de la bibliografía anterior (Vallespí, 1986); en la misma obra se añaden aportaciones directas de Gibert sobre la atribución antropológica de Orce y de Botella, Martínez y Cárdenas sobre la base achelense de Cueva Horá.

Desde esa fecha es importante la documentación acumulada sobre el Paleolítico Inferior de la región, sustancialmente referida, en lo que ahora nos atañe de industrias achelenses, al planteamiento de una secuencia de Achelense y Paleolítico Medio en las terrazas fluviales de la Depresión Inferior del Guadalquivir (recogida en su mayor parte en Díaz del Olmo y Vallespí, ed. 1988; Díaz del Olmo y Rodríguez Vidal, ed. 1989) y a la revisión crítica de Cúllar de Baza I y La Solana de Zamborino (Vega Toscano, 1989).

Sobre la Depresión Inferior del Guadalquivir, el desglose de trabajos de las citadas obras colectivas de 1988 y 1989 presenta aportaciones de contenido estratigráfico de Castiñeira, García Rincón, Alvarez y Martín; Santoja y Querol (texto inédito de 1976); Díaz del Olmo y Vallespí; Vallespí, Díaz del Olmo, Alvarez y Vallespí García; y Giles y Santiago; todos ellos en la publicación de 1988, y en la de 1989, Díaz del Olmo, Vallespí, Baena y Recio; Giles, Santiago, Gutiérrez, Mata y Aguilera; Castiñeira, Alvarez, García Rincón, Gómez, Martín y Rodríguez Vidal; Ramírez Delgado, Fernández-Llebrez y Mateos; y sobre materiales exclusivamente de superficie, trabajos en 1988 de Pereda y Martínez; Rodríguez Tamiño; Vallespí y Amores. A series de superficie se refieren, desde otras publicaciones, las aportaciones sobre esta región del Bajo Guadalquivir de Buero, Castiñeira y García Rincón, 1985; Castiñeira y García Rincón, 1985; Araque y Ruíz, 1986; Monclova y Giles, 1987; Araque, 1987; García Rincón, 1987; Vallespí, 1987; Asquerino, 1988; y Giles y Santiago, 1988. Fuera de la Depresión Inferior del Guadalquivir, al tramo medio-alto del Genil se refiera la aportación de Toro Moyano y Ramos Lizana en 1988. A todo ello cabe añadir, desbordando lo estrictamente achelense que consideramos, lo publicado en dos importantes obras colectivas, sobre la Cuenca de Guadix-Baza, dirigida por Alberdi y Bonadonna, y sobre Orce, por Gilbert, Campillos y García Olivares, ambas publicaciones de 1989.

A ello hay que añadir mis planteamientos teóricos sobre las culturas de graveras y el arranque achelense y sobre la perduración postachelense en el Palelítico Medio, en su perspectiva peninsular (Vallespí, 1986, 1988 y 1989), y finalmente, un último trabajo de síntesis (Vallespí, e.p.).

Ante tal cúmulo de títulos recientes, cuya valoración global resulta sin duda muy positiva, conviene empero aclarar, para evitar cualquier juicio apriorístico engañoso, que en su mayor parte se trata de informes y de avances, entre los que hay además excesivas entregas reiterativas, sin entrar en la dialéctica de la propia investigación que marcan las líneas de trabajo del proyecto al que se implican, y que faltan todavía los estudios detallados, que a corto plazo deberemos disponer.

\section{RELACION Y BIBLIOGRAFIA DE YACIMIENTOS Y HALLAZGOS}

Desde la referencia de los primeros hallazgos andaluces en el corpus de la segunda edición de $E l$ Hombre Fósil de Obermaier, en 1925, las recopilaciones de los yacimientos achelenses de la región han estado relegados a las obras generales referidas a la totalidad de la Península Ibérica, en particular 
las de Pericot, Epocas primitiva y romana, en Historia de España. Gran Historia General de los Pueblos Hispanos, de 1934, y de Almagro, El Paleolítico, en Historia de España, dirigida por Menéndez Pidal, de 1947, y recientemente a las visiones en manuales, de Santonja y Querol, Prehistoria, en el colectivo Manual de Historia Universal de 1983, y de Jordá, Prehistoria, en la también obra colectiva Historia de España, de 1986, hasta mi citado trabajo de dicho año 1986, que constituye la primera visión regional del Paleolítico Inferior y Medio de Andalucía, donde se actualiza la relación de todos los hallazgos con sus referencias bibliográficas, en un planteamiento de síntesis de sus grandes líneas interpretativas (Vallespí, 1986).

Los avances realizados desde esa fecha, expuestos en la reseña historiográfica precedente, alteran el panorama achelense de la región de manera sustancial, al demostrar la existencia de industrias estratificadas en los depósitos fluviales de la Depresión Inferior del Guadalquivir anteriores al horizonte avanzado entonces conocido de La Solana del Zamborino y Cueva Horá, lo que permite el planteamiento de una secuencia regional, que propongo en mi trabajo últimamente entregado (Vallespí, e.p.). $\mathrm{Al}$ mismo tiempo, parece evidenciarse una fuerte perduración achelense en el Paleolítico Medio, que, a mi modo de ver, en los ámbitos fluviales de la Baja Andalucía desborda el sentido limitado de las facies musterienses de tradición achelense, para constituirse en un Paleolítico Medio postachelense, de sentido no clásico, lo que visto desde otro ángulo vendría a suponer también la aparición temprana, en plenos achelenses de la región de lo que podríamos denominar el fenómeno tecno y tipológico musteriense. La realidad es que existe una dificultad de distinción, en la práctica, entre series achelenses avanzadas y determinadas series del Paleolítico Medio de la región. Esta continuidad del Achelense Superior y Final y el Paleolítico Medio de aspecto postachelense parece evidenciarse en la secuencia del Bajo Guadalquivir, sobre cuya experiencia he intentado fundamentar estratigráficamente mi hipótesis de trabajo, planteada en publicaiones recientes (Vallespí, 1988 y 1989).

Como se trata de trabajos de campo en curso de realización y de estudios analíticos sin publicar, dados a conocer únicamente en avances e informes valorativos, sin tiempo hasta el momento para su registro bibliográfico en las visiones críticas últimamente publicadas (Vega Toscano, 1989, con bibliografía incorporada de estas tareas ínferopaleolíticas hasta 1986, y Santonja y Villa, 1990, hasta 1982), parece oportuno presentar ahora la relación individualizada de yacimientos y hallazgos, previamente a su ordenación en un panorama conjunto, que aspira además a estimular su estudio directo en el campo de trabajo del ámbito territorial andaluz.

Por las razones antedichas, en la elaboración de la lista que presento he seguido el criterio flexible de incluir, junto a los yacimientos y localizaciones con fundamentación estratigráfica, pertenecientes a los complejos con bifaces, hendedores y triedros, calificables por ello de achelenses (con toda la relativización que se quiera de este término), la relación de la series de superficie con bifaces, hendedores y triedros y los hallazgos sueltos de bifaces, genéricamente atribuibles a la línea achelense y Paleolítico Medio postachelense, relegando su discusión valorativa al texto del estudio. La relación que presento no contempla, por lo tanto, a los restantes yacimientos del Paleolítico Inferior de la región carentes de las evidencias tipológicas referidas. No entran tampoco en esta lista una serie de noticias pendientes de verificación, ni las novedades inéditas de varias tesis doctorales en realización con mi responsabilidad académica. Algunas otras exclusiones, de atribuciones dudosas o infundadas, se indican más adelante. Con todo ello, la relación resultante de yacimientos, localizaciones y hallazgos, con su apoyo bibliográfico básico o referencial, no exhaustivo, es la siguiente: 
1. Yacimientos y localizaciones en estratigrafía

1.1. Bajo Guadalquivir:

1.1.1. Alrededores de Ecija: Vallespí, e.p.

1.1.2. Arroyo del Rubio: Reseñado con esta denominación y con anterioridad como Paleocanal de San Pedro y T4P (Terraza 4, Paleocanal, en Díaz del Olmo y Vallespí, 1988a: 54, y 1988b: 46 y lám. en p. 45; Díaz del Olmo, Vallespí y otros, 1989: 41; y Vallespí, e.p.

1.1.3. Tarazona, con dos localizaciones cercanas: I, Díaz del Olmo y Vallespí, 1988a: 54 y 1988b: 48-49; Vallespí, e.p.; y II, Vallespí, e.p.

1.1.4. Las Jarillas y Antiguos Viveros, lugares cercanos en el mismo nivel: Díaz del Olmo y Vallespí, 1988a: 54; y 1988b: 51-52; Vallespí, e.p.

1.1.5. Localizaciones menores, abundantes en todo el ámbito estudiado (sectores La Campana y Carmona de las terrazas del Guadalquivir y afluentes secundarios y terrazas de la margen derecha, con algunas referencias en Vallespí y otros, 1952; y Díaz del Olmo, Vallespí y otros, 1989: 37-41).

1.2. Guadalete:

1.2.1. Laguna de Medina: Giles y otros, 1989a.

1.2.2. Palmar del Conde: Giles y otros, 1990.

1.2.3. Otras localizaciones: Giles y otros, $1989 \mathrm{~b}$.

1.3. Tinto:

1.3.1. El Monturrio: Rodríguez Vidal y otros, 1988: 21-23; Castañeira y otros, 1989: 62.

1.3.2. La Glorieta: García Rincón, 1985; Rodríguez Vidal y otros, 1988: 23-28; Castiñeira y otros, 1989: 61-62.

1.4. Cuenca de Guadix-Baza y su reborde occidental:

1.4.1. Solana del Zamborino: Botella y otros, 1976; Vega Toscano, 1989: 333-337 y ss.

1.4.2. Cueva Horá, niveles infeiores: Botella y otros, 1986.

2. Localizaciones en superficie.

2.1. Alto Guadalquivir:

2.1.1. Puente Mocho: Cabré y Wernert, 1916; Carrasco y otros, 1979.

2.1.2. Cortijos Piedras de Santa Inés y Angulo: Sánchez Martínez y García García,1977.

2.2. Guadalquivir medio y tramo medio-alto del Genil:

2.2.1. Terraza del Guadajoz: Carbonell, 1931; Casas Morales, 1964; Beguiristáin, 1976.

2.2.2. Cortijo del Calvillo: Carrasco y otros, 1986; Toro Moyano y Ramos Lizana, 1988.

2.2.3. Arroyo del Ventogil: Araque, 1987.

2.3. Bajo Guadalquivir:

2.3.1. Arroyo de la Víbora: Amores, 1982; Vallespí y otros, 1982; Vallespí y Amores, 1988.

2.3.2. Villaesparra: Amores, 1982; Vallespí y Amores, 1982.

2.4. Depresión de la Janda: Loma del Machorro: Breuil, 1914; Hernández Pacheco, 1915; Breuil, 1917; Giles y Sáez, 1980; Fernández-Llebrez y Mateos, 1989.

3. Hallazgos sueltos de bifaces.

3.1. Río Blanco, en la Sierra Sur de Sevilla, término de los Corrales: Vallespí, 1987.

3.2. Confluencia del Cabra con el Genil, término de Santaella (Córdoba): Vallespí, Cruz-Auñón y Rivero, e.p.

3.3. Terraza del Guadalquivir, término de Dos Hermanas (Sevilla): Vallespí y Amores, 1988.

3.4. Terraza del Sábar, en el Alto Vélez (Málaga): Vallespí, Ramos Muñoz y Martín Córdoba, 1989-90. 


\subsection{Playa de La Barrosa, término de Chiclana de La Frontera (Cádiz): Vallespí, Escacena y Ramos Muñoz, e.p. \\ 3.6. Ría del Tinto, término de Lucena del Puerto (Huelva): Vallespí, Del Amo y Alvarez, 1981.}

Esta relación tiene, como ya he dicho, toda la provisionalidad que le impone el incipiente conocimiento actual de los yacimientos ínferopaleolíticos de la región. Con respecto a las dificultades que suponen la falta de datos y algunas veces de garantías en las atribuciones publicadas, cabe exponer algunas observaciones:

De los yacimientos con fundamentación estratigráfica anotados, los dos únicos que han sido objeto de excavación, La Solana del Zamborino y Cueva Horá, lo han sido sólo con excavación parcial, el primero de ellos sin continuidad en las tareas y ambos limitados en su pubblicación a sendos informes, sin las memorias de lo realizado (cfs. sobre el primero la reciente crítica de Vega Toscano, 1969: 333337). Los demás yacimientos están todos prácticamente sin publicar, reducidos bibliográficamente a las noticias de su control y la valoración de su posición geomorfológica en sus respectivas secuencias fluviales y de las muestras de industrias extraídas de los depósitos detríticos, en diversos informes; alguno incluso ha sido descubierto últimamente. No obstante aparece con suficiente fundamento geomorfológico y arqueológico la atribución al achelense de estos yacimientos incluídos en la relación, a salvo, claro está de rectificaciones, siempre posibles, y de lo que resulte sobre la noticia adelantada de la localización del Guadalhorce, de momento sin su contexto documentado. La matización que ofrezco de localizaciones menores se refiere, en los medios fluviales, a los perfiles de terrazas de escaso control de piezas arqueológicas extraídas, en contraste con su concentración en conjuntos correspondientes a verdaderos yacimientos, desplazados o incluso in situ en sentido algo flexible, hasta su verificación definitiva.

Con respecto a las series de superficie, hay que advertir primeramente que la lista presentada no recoge algunas localizaciones con piezas descritas como bifaces pero que, en sus dibujos, me parecen de dudosa morfología y por ende, provisionalmente descartables, e incluso en algún caso impropiamente clasificadas como tales piezas, claramente identificables con otros tipos en sus publicaciones, por lo que resultan desechables, hasta su revisión, las atribuciones de los correspondientes yacimientos. Estos yacimientos de superficie, con sus series sobre las que recae especialmente, como es obvio, la incertidumbre metodológica de la mixtificación de las industrias y la dificultad comentada de distinción entre series achelenses avanzadas y del Paleolítico Medio postachelense, presentan en la lista establecida conjuntos achelenses incuestionables y representativos de la amplitud de estas series en la región, aunque conocidos únicamente por informes iniciales, junto a otros claramente posteriores, por lo que la reserva metodológica los afecta en diverso grado, si bien quedan todos pendientes de un estudio acabado. Su aclaración definitiva no será posible hasta que sean mejor conocidos y además dispongamos de apoyos estratigráficos generales e inmediatos en la región, posiblidad ésta última que parece vislumbrarse en algunos de los yacimientos registrados en el Bajo Guadalquivir. Otro tanto cabe decir de los hallazgos sueltos de bifaces, con tipos clásicos de diagnóstico tipológico aceptable, sobre cuyo interés de su publicación he insistido en varios trabajos, aunque algunos queden sin más precisión que la flexible línea achelense superior y paleolítico medio a la que corresponden, y además, con generalización de cantos de talla muy desarrollada bifacial parcial, comunes a todo el desarrollo de esos complejos industriales e inutilizables para un diagnóstico. La limitación de la lista que presento obedece al criterio de incluir sólo los publicados de atribución fundamentada en su adecuado análisis. A otro punto de vista corresponde la observación de que hasta el momento son los hallazgos sueltos de bifaces, y no los yacimientos de superficie, como hay que esperar, los que presentan la única evidencia 
controlada de la presencia humana en terrenos de formación precuaternaria (con el sólo ejemplo disponible del hallazgo de Los Corrales, en laderas del tramo subbético occidental sevillano), lo que apunta a un vacío en las prospecciones, que hay que superar para el conocimiento de la dispersión territorial achelense.

\section{PANORAMA DE LAS INDUSTRIAS: PLANTEAMIENTO ACTUAL Y PERSPECTIVAS}

Sobre el Paleolítico Inferior de Andalucía disponemos tan sólo de dos breves visiones de conjunto, en sendos trabajos míos, el publicado en 1986 y un segundo entregado en 1990 y en prensa. En la primera ordenación, expuesta en el Congreso de Homenaje a Luis Siret, celebrado en junio de 1984 y publicadas sus actas dos años después, quedó planteada mi hipótesis de vertebración de las industrias regionales en unas culturas iniciales de las graveras, el Achelense con ellas relacionado, que desbordaría en momentos avanzados su contexto inicial de las graveras, y una perduración de estos complejos industriales, con impacto musteriense, en un Paleolítico Medio diferenciado de los modelos clásicos. Con respecto al Achelense, ante la inviable aplicación de las subdivisiones clásicas al registro regional conocido, consideré la hipótesis simplificadora de un posible Achelense Antiguo (equivalente al Inferior y Medio primitivo clásicos), sin testimonios controlados en la región, y un Pleno Achelense (Medio evolucionado y Superior tradicionales), comprendiendo toda la documentación andaluza conocida hasta el momento (Vallespí, 1986: 59-62).

Faltaba entonces la fundamentación estratigráfica de estas series achelenses de la región anteriores a La Solana del Zamborino, en un vacío del pleno Pleistoceno Medio al que se refirió Vega Toscano con una rigurosa valoración crítica en 1989 y sobre cuyo contenido arqueológico íbamos aportando mientras tanto las primeras evidencias coherentes, con la estructuración de una secuencia geomorfológica y arqueológica en el Bajo Guadalquivir (Díaz del Olmo y Vallespí, 1988 y 1989; Díaz del Olmo, Vallespí y otros,1989). Al mismo tiempo perfilaba mi teoría, ya formulada en 1986, del carácter postachelense del Paleolítico Medio en dichos medios fluviales de la región, configurado en un ámbito peninsular más amplio (Vallespí, 1988 y 1989).

Todas estas aportaciones y otras novedades recientes que han quedado reseñadas en el balance historiográfico precedente, requerían su sistematización en una nueva síntesis regional, que obviara al mismo tiempo la dispersión de los datos y algunas imprecisiones en su atribución, y a ello he dedicado la atención requerida en una valoración bibliográfica de los avances del Paleolítico y Epipaleolítico de Andalucía desde 1986, últimamente presentada y de publicación inminente, donde expongo mi particular visión conjunta actualizada del Achelense de la región. El estudio plantea la integración de todos los registros hasta ahora conocidos en el Pleno Achelense de sentido convencional achelense medio evolucionado, superior y final, sin control garantizado por el momento de testimonios achelenses más antiguos y con perduración evidenciada en el Paleolítico Medio, especialmente en los ámbitos fluviales de la Baja Andalucía, donde constituye un Paleolítico Medio postachelense de corte no clásico; las formaciones fluviales de la Depresión Inferior del Guadalquivir y los depósitos de La Solana del Zamborino y de la base estratigráfica de Cueva Horá permiten plantear la vertebración cronoestratigráfica, en la segunda mitad del Pleistoceno Medio y su transición, de esta secuencia achelense regional (Vallespí, e.p.).

Esta última interpretación sirve de base al presente panorama, que añade a su texto las ampliaciones pertinentes y algunas reflexiones sobre las perspectivas inmediatas del estudio. 


\subsection{Los indicios y evidencias iniciales}

A escala estrictamente regional, lo primero que cabe plantearse es la relación del Achelense posterior con los indicios y evidencias iniciales del poblamiento humano en la región: indicios de Orce, testimonio de Cúllar de Baza I y atribuciones por verificar en las terrazas altas del Bajo Guadalquivir, apartado como queda el Aculadero de una datación tan antigua.

El conocimiento de Orce sigue en su impasse arqueológico, aunque enriquecido con nuevas atribuciones de los lugares circundantes de Cortijos de Doña Milagros, con un chopper de cuarcita y dos lasquitas de sílex, y Cortijo de D. Alfonso, con una lasca de sílex, relacionadas al parecer con los depósitos (Gibert y otros, 1989). La evidencia arqueológica indiscutible de Cúllar de Baza I ha aumentado últimamente con el registro de dos lascas de sílex especialmente frescas (Vega Toscano, 1989: 331-332). La posiblidad de atribución al Pleistoceno Medio Antiguo de evidencias de cantos tallados en la terraza alta del Guadalquivir, en Cerro Higoso, queda pendiente de verificación, pues a las evidencias recogidas inicialmente de una industria en posición cronomorfológica dudosa hay que añadir la de un canto con filo unifacial de intencionalidad discutible, del interior del muro del depósito de la terraza, de segura atribución. El descabalgamiento, en cambio, de datación tan antigua para el yacimiento de El Aculadero, por demás razonable, dados los argumentos geológicos y la propia reconsideración de su complejo lítico (Zazo y otros, 1985; Santonja y Villa, 1990), limita los precedentes del Achelense de la región a estos cortos, pero importantes registros señalados, abiertos todavía a su verificación, excepto el caso inequívoco de Cúllar de Baza I.

Sobre esta corta, aunque importante, documentación poco puede pensarse con respecto a su relación con las posteriores industrias achelenses de la región, de las que por el momento queda separada por un hiatus entre los comienzos del Pleistoceno Medio de Cúllar de Baza I y en su caso, de Cerro Higoso, hasta el inicio de la seriación achelense del Guadalquivir inferior del Pleistoceno Medio pleno y avanzado, que teóricamente afectarían a las cuestiones del Achelense Antiguo y de unas precedentes industrias arcaicas de cantos tallados. Tan sólo cabe señalar a estos respectos que cantos tallados, con alguna relación con las graveras, son los soportes del chopper del Cortijo de Doña Milagros de Orce y de varias, dos al menos, piezas de Cúllar de Baza I, así como del canto de filo unifacial de talla siscutible de Cerro Higoso, lo que permite entrever cierta relación con las culturas iniciales de las graveras y la hipotética génesis de un Achelense Antiguo autóctono, de mi teoría (Vallespí, 1988), cuya argumentación se enriquecería sensiblemente en el caso de confirmarse la validez de la atribución de la industria de cantos tallados, y quizás con algún bifaz, del techo del perfil de la terraza de Cerro Higoso, aunque de momento no podamos argumentar sobre supuestos pendientes de seria verificación. De este modo, las evidencias reseñadas disponibles por ahora, sin otros testimonios aceptables, dejan en puro planteamiento teórico la cuestión de las industrias arcaicas de cantos tallados y del Achelense Antiguo de la región andaluza, aunque queda positivamente abierta su investigación.

\subsection{Las industrias achelenses y sus bases estratigráficas}

Tras el vacío arqueológico señalado, la seriación achelense regional aparece actualmente constituída por las industrias del interior de los depósitos de terrazas de la secuencia fluvial del Bajo Guadalquivir y Guadalete y Tinto, en Andalucía occidental, y por los yacimientos de La Solana del Zamborino y la base estratigráfica de Cueva Horá, en la Alta Andalucía de la Cuenca de Guadix-Baza y reborde, a cuya vertebración se suman las series de superficie de más amplia distribución espacial. A mi modo de ver, todo lo controlado hasta ahora de este Achelense regional debe cubrir el Pleistoceno Medio 
Reciente y su transición al Superior y prefigura un Achelense pleno, en el sentido de Achelense Medio evolucionado y sobre todo Superior, con un estadio final, transicional a una perduración postachelense en el Paleolítico Medio, bien patente en los ámbitos fluviales de la Baja Andalucía. (Usando dichas subdivisiones arqueológicas achelenses en su sentido convencional empleado hasta ahora).

La investigación de las terrazas del Guadalquivir Inferior fue iniciada por mí y G. Alvarez en el curso académico 1977-78, integrada como trabajo de mi investigación personal en el Programa de Fomento de Investigación del Ministerio de Investigación y Ciencia en los cursos 1980-81 y 1981-82, y convertida desde 1985, con la codirección de F. Díaz del Olmo y la participación de colaboradores de Arqueología y Geomorfología, en Proyecto de Investigación de la Dirección General de Bellas Artes (después, de Bienes Culturales) de la Consejería de Cultura de la Junta de Andalucía, con publicaciones desde 1982. Las tareas geoarqueológicas realizadas hasta el presente han permitido la distinción de 16 niveles de terrazas pleistocenas y la fijación de una seriación de conexiones de industrias con los depósitos de las terrazas medias y bajas, del siguiente modo: indicios ya reseñados en T-V y T-VI; TVII y T-VIII, arqueológicamente estériles; T-IX con conexiones de muestras de industrias; T-X, estéril; y una seriación ininterrumpida de muestras y conjuntos de conexiones de industrias con los depósitos desde T-XI a T-XV, ambas inclusive, aparte de T-XVI, del Pleistoceno Reciente-Holoceno, con algunas pocas (Díaz del Olmo, Vallespí y otros, 1989).

La mayor parte de estas conexiones estratigráficas de industrias se limita a pocas piezas dispersas en los depósitos detríticos, como localizaciones menores, pero en algunos casos se trata de concentraciones de piezas constituyendo conjuntos industriales correspondientes a yacimientos. A las series achelenses corresponden el yacimiento de Arroyo del Rubio, la localización de la Celada, los yacimientos de Tarazona, Antiguos Viveros y Las Jarillas, en terrazas del Guadalquivir del sector Carmona, la localización del Arroyo Viñuela, en el sector La Campana, y un yacimiento en los alrededores de Ecija, en el Genil inferior, últimamente descubierto y pendiente de verificación, todos ellos con muestras de industrias con cantos tallados, bifaces, hendedores y triedros y tipos sobre lasca de la lista normativa, extraídas del interior de los depósitos.

La posición cronomorfológica de estos yacimientos, entre los niveles T-IX y T-XIII de la secuencia fluvial, presenta cuestiones por aclarar, referentes sustancialmente a la posición de los yacimientos del Arroyo del Rubio, en un paleocanal, de Tarazona I y II, en el sector Carmona, y a la corelación con dicho sector de la terraza del yacimiento del Arroyo Viñuela, en el sector La Campana, y del yacimiento de los alrededores de Ecija, en el Genil, aparte de cuestiones menores en algún otro yacimiento. Pero apreciada en su conjunto, la serie de estos yacimientos achelenses cubre el Pleistoceno Medio reciente y arqueológicamente confirma un Achelense susceptible de precisiones diacrónicas aún por determinar, correspondiente a lo que, para el ámbito andaluz, he calificado (Vallespí, 1986: 60) de Achelense Pleno y que en términos convencionales clásicos equivaldría tal vez al Achelense Medio evolucionado y con seguridad al Achelense Superior y Final, este último estadio bien determinado en la secuencia por la concurrencia de los datos geomorfológicos, paleontológicos y arqueológicos en un nivel, T-XIII, que consideramos transicional a las terrazas subyacentes.

De modo que, tanto por las bases morfoestratigráficas establecidas como por el aspecto apreciable de sus industrias, en dicha seriación se perfilan claramente dos horizontes culturales: el Achelense calificable globalmente de Superior de las terrazas medias, representado por los yacimientos y localizaciones de Arroyo del Rubio, La Celada, Arroyo Viñuela, Tarazona I y Ecija, y el Achelense Final del nivel que comienza las terrazas bajas, con los yacimientos de Antiguos Viveros y Las Jarillas, en continuidad transcional del Paleolítico Medio, que he calificado de postachelense, de dichos niveles bajos. En el estado actual de la investigación, culminada la primera fase programada para planteamien- 
to de la secuencia geomorfológica fluvial y fijación de las conexiones estratigráficas de industrias, contamos sólo con las muestras extraídas de los cortes de perfiles revisados, cuyo cuadro provisional conjunto presenta un bloque común de cantos tallados, bifaces, hendedores y picos triédricos, bien representado en el Achelense Superior y reducido en el Final, y por el bloque de tipos de la lista normativa sobre lasca, simplificado al principio y con notable desarrollo en tipos y modos de retoque de los Grupos Levallois y Musteriense, y más desdibujadamente el Grupo Paleolítico Superior, en el horizonte final, inseparable, por ello del subsiguiente Paleolítico Medio de los niveles bajos de la secuencia.

A estas series achelenses corresponde también el yacimiento de La Laguna de Medina, en la cuenca media-baja del Guadalete, con materiales procedentes del interior de la terraza a $-30-50 \mathrm{~m}$. sobre el curso actual del río, con cantos tallados, bifaces, hendedores y triedros y algunos tipos sobre lasca de la lista normativa, que a mi modo de ver encajan perfectamente en mi concepción del Pleno Achelense de la región, y concretamente en su horizonte Medio evolucionado y Superior convencional, más bien en este último estadio (Giles y otros, 1988 y 1989). Fuera de la Baja Andalucía, se ha atribuído recientemente a estas mismas series un conjunto de materiales en conexión estratigráfica de Aljaima, en el bajo valle del río Guadalhorce, en una nota de avance de su publicación (Barroso y otros, 1989).

Además de los yacimientos y localizaciones reseñados, todos los cuales presentan algunas muestras de bifaces, hendedores y triedros entre los materiales extraídos del interior de los depósitos detríticos, hay algunos otros que por su posición geomorfológica deben corresponder a los mismos complejos achelenses, aunque circunstancialmente falten en sus muestras extraídas ejemplares de dicho bloque tipológico diagnóstico, como es el caso de una serie de localizaciones menores del Bajo Guadalquivir, como Gastaembalde, Nuestra Señora de Gracia, Sinergiprón, San Germán y Calongo Alto, y parecen serlo también el nivel III de La Glorieta (Rodríguez Vidal y otros, 1988: 28; Castiñeira y otros, 1989: 61-62) y El Monturrio (Castiñeira y otros, 1989: 62), en terrazas del Tinto, y en la cuenca media-alta del Guadalete, la localización del sector del Pantano de.Bornos (Giles y otros, 1989: 45). Se trata, en suma, de un pleno Achelense de graveras con fundamentación estratigráfica en las formaciones fluviales del Pleistoceno Medio Reciente y comienzos del Superior de la Baja Andalucía y extensiones análogas y cuyo arranque en dichos ámbitos de la región es sin duda más antiguo, aunque, como he comentado, permanece sin aclarar.

Fuera de los medios fluviales el Achelense aparece en momentos algo avanzados. En la Depresión de Guadix-Baza, el importante yacimiento de La Solana del Zamborino puede estar, en efecto, en relación con el horizonte avanzado representado por alguno de los conjuntos señalados, pues parecen coincidir en presentar el mismo problema de constitución de unos complejos industriales con bifaces, hendedores y picos triédricos, en el Guadalquivir, junto a tipos sobre lasca de la lista normativa muy evolucionados, con aspecto tecno y tipológico musteriense en pleno Pleistoceno Medio avanzado, aunque conviene esperar la comprobación crono-estratigráfica rigurosa de los yacimientos implicados. La cuestión puede tener alcance peninsular, dentro de las nuevas perspectivas de su planteamiento en el marco occidental clásico; en tal sentido y a la vista de los materiales del Guadalquivir, recogí recientemente la opinión oral de M. Santonja. El yacimiento del Guadalquivir al que nos referimos especialmente, Tarazona II, descubierto últimamente, está en vía de estudio y de Solana del Zamborino no hay más noveedad que la discusión crítica de Vega Toscano, 1989: 333-337, a la que referimos nuestra reflexión.

La atribución al Achelense Superior meridional de la base de Cueva Horá (Botella y otros, 1986) puede tener también alguna relación con estas cuestiones, cuya discusión, insisto, resulta ciertamente prematura. Lo que no hace falta resaltar en este caso es el interés de la comprobación de la existencia 
de industrias achelenses en el arranque de una estratigrafía musteriense en cueva de nuestra región, hecho desconocido hasta ahora, y al que puedo asociar, con las debidas reservas, mi impresión de los materiales inéditos que conozco de una cueva cordobesa, Cueva del Angel, en Lucena, con varios bifaces de aspecto achelense superior y musteriense y series musterienses sobre lasca, procedentes al parecer del abrigo de entrada de la oquedad y que parecen apuntar en el mismo sentido que comentamos. Pero todo esto se implica también en la cuestión de mi planteamiento de un Paleolítico Medio de carácter postachelense en la región, que expongo luego.

La extensión territorial y ciertas densificaciones de focos locales de este Achelense Pleno y Final aparecen matizadas con la distribución de las series de superficie conocidas, en las que no siempre es posible la distinción de conjuntos en la línea diacrónica del Achelense Superior a la perduración postachelense en el Paleolítico Medio. De la relación de localizaciones presentadas en mi primera síntesis de 1986, ofrecen ejemplares de bifaces, hendedores y triedros los materiales publicados de la Depresión de la Janda, las terrazas del Bajo Guadalquivir de la provincia de Sevilla y del Guadajoz en Córdoba, y el amplio foco del Alto Guadalquivir en la provincia de Jaén, desde el Guadalimar en Puente Mocho hasta el río Jándula (cfs. la bibliografía en Vallespí, 1986: 60), a las que se añaden las aportaciones recientes: en la Depresión Inferior del Guadalquivir, Arroyo de Ventogil (Araque, 1987), otras localizaciones en la provincia de Córdoba (Asquerino, 1988; Araque y Ruíz Gómez, 1989), terrazas de la provincia de Sevilla (Vallespí y otros, 1988; Pereda Acién y Martínez Rodríguez, 1988; Vallespí y Amores, 1988); río Guadalete (Giles y Santiago, 1988; Giles y otros, 1989); provincia de Huelva (Castiñeira, García Rincón y otros, 1988); La Janda (Ramírez Delgado, Fernández-Llebrez, Mateos, 1989); tramo Subbético sevillano (Vallespí, 1987); y en la tierra de Loja, el Cortijo del Calvillo (Toro Moyano y Ramos Lizana, 1988).

\subsection{Las industrias postachelenses del Paleolítico Medio}

Según mi interpretación actual, en los grandes ámbitos fluviales de la región el proceso de las industrias achelenses de los medios de graveras se desarrolla como un contiuum de explotación para la talla de sus recursos de cantos rodados, desde un arranque muy antiguo todavía por determinar y sin solución de continuidad hasta avanzados los tiempos del Pleistoceno Superior, cubriendo al menos su primera mitad y desbordándola muy posiblemente, lo que, de confirmarse rompería el esquema clásico de una diferenciación tan marcada como ahora se supone entre los casilleros arqueológicos convencionales del Paleolítico Inferior y Medio y en alguna medida también con el Paleolítico Superior Antiguo.

Esta hipótesis de la perduración de las industrias ínferopaleolíticas en el Paleolítico Medio de los ámbitos fluviales de Andalucía occidental quedó expuesta en mi primera síntesis y ha sido estructurada en recientes trabajos, ordenando los datos morfoestratigráficos y arqueológicos de la Depresión Inferior del Guadalquivir, que permiten fundamentar tal interpretación, e intentando plantear su alcance peninsular (Vallespí, 1988 y 1989).

Según mi interpretación, en los ámbitos fluviales de la Depresión Inferior del Guadalquivir, con su proyección al Guadalete y al Tinto-Odiel, aparece un Paleolítico Medio claramente diferenciado de los complejos musterienses clásicos, de cuevas, en ambientes de montaña, y su diferenciación radica en el aspecto postachelense de las industrias de las formaciones fluviales, generadas en la tradición de las graveras. Hay, por lo tanto, que distinguir este Paleolítico Medio postachelense, que no cabe seguir dominando musteriense, de los complejos musterienses, a los que quedaría restringido el sentido habitual de las facies, que perdería de este modo su vigencia generalizada. En su distribución en la región, ambos modelos de ocupación territorial reflejarían las diferencias geográficas, ambientales y en 
recursos, de Andalucía Alta y Baja, con los matices y mixtificaciones que pueden previamente suponerse y, en su caso, se demuestren.

En la secuencia regional de la Depresión Inferior del Guadalquivir, las industrias del Paleolítico Medio están representadas por las muestras extraídas del interior de los depósitos en las localizaciones de las terrazas bajas, T-XIV y T-XV (Morilla, Camino 24-25, San José de la Rinconada, Brenes, Toruño y Guadajoz), en la margen izquierda del sector Carmona y en otras localizaciones de la margen derecha (Peones Camineros, Santa Iglesia, San José y Arroyo Gabino), en una localización de la terraza baja del Guadalete, del sector de Villamartín (Vallespí y otros, 1983-84; Giles y otros, 1989: 45), y en sendaas localizaciones (Apeadero de Niebla y el Gritillo) en el Tinto y Odiel (Castiñeira y otros, 1989: 65). Carentes de momento las localizaciones de Huelva y Cádiz de estructuración de las secuencias fluviales sustentantes, la argumentación de mi hipótesis debe por ahora basarse en la secuencia del Guadalquivir.

En las terrazas del Bajo Guadalquivir, el-Paleolítico Medio aparece, en continuidad con la seriación achelenses y tras un nivel achelense final transicional, como un complejo terminal del proceso de las industrias, definido por un doble componente: la persistencia de un fuerte sustrato arcaizante y el novedoso impacto musteriense. El sustrato está condicionado por la constante secuencial de la talla exclusiva sobre cantos rodados, con sus reflejos tecno y tipológicos limitadores, y aparece definido tipológicamente por la continuidad generalizada de los cantos tallados, choppers más que choppingtools, y la limitación de los tipos sobre lasca, reiterando tipos de morfología ínferopaleolítica, tales como raederas simples y cuchillos de dorso natural o atípico. De momento, falta en las muestras extraídas el bloque macrolítico de bifaces, hendedores y pico triédricos, aunque de los primeros hay ejemplares del interior de depósitos por determinar, así como de superficie asimilables. El impacto musteriense se traduce en la generalización de la talla levallois de lascas y la presencia de puntas, la abundancia de núcleos específicamente musterienses y una mayor atención al retoque, con los nuevos modos característicos (escamoso y escalariforme) y matizaciones tipológicas enriqueciendo al Grupo Musteriense (raederas dobles, convergentes, transversales, y algún otro tipo) y escasamente al Grupo Paleolítico Superior (perforadores y apenas otros tipos) y suponiendo también el aumento de algunos tipos comunes, como muescas y denticulados, además de una abundancia de Diversos. Asimismo destacable es el aumento selectivo de la talla de sílex, pasando, en las muestras recogidas, aproximadamente del $24 \%$ del Achelense Final al $40 \%$ en el Paleolítico Medio, que queda de este modo más equilibrado con el empleo de cuarcitas. Todo ello en una mera prefiguración inicial de las industrias (Vallespí, 1988; y 1989: 8-10 y 16), hasta que queden estudiados debidamente todos sus materiales y se aclare la posicicón morfoestratigráfica del conjunto de Tarazona II, en una de ambas hipótesis: en el Achelense avanzado, tal como lo hemos comentado anteriormente, o ya en el Paleolítico Medio. Recordando la riqueza de este conjunto de Tarazona II (en bifaces, hendedores y triedros y en los Grupos Musterienses y, en menor grado, Paleolítico Superior), captaremos el interés que supone su definitiva datación relativa para la comprensión de la secuencia y de las cuestiones que ahora comentamos.

\section{BIBLIOGRAFIA}

AGUIRRE, E.; COLLINS, D. y CUENCA, J. (1962): "Perspectivas del Paleolítico Inferior en España", Noticiario Arqueológico Hispánico VI, cuad. 1-3: 7-14.

AMO, M.del. (1976): "Restos paleolíticos y neolíticos en la provincia de Huelva", Huelva Arqueológica, II, p.191-196, lam. Publicaciones del Museo de Huelva. 
AMORES, F. (1982): Carta Arqueológica de los Alcores (Sevilla). Sevilla Diputación Provincial.

ARAQUE, F.A. (1987): "Paleolítico Antiguo en la provincia de Córdoba. El Arroyo del Ventogil (Fernán Núñez), Estudios de Prehistoria Cordobesa,3, p.3-17.

(1987): "Prospecciones arqueológicas superficiales en los valles del Guadajoz y Guadalquivir, provincia de Córdoba, 1985-86, Anuario Arqueológico de Andalucía,1986, II, p. 12-18.

ARAQUE, F; RUIZ, A, M. . . (1986): "Trance Pajares: un yacimiento paleolítico en la provincia de Córdoba, Estudios de Prehistoria Cordobesa, n. 1, p.3-20. Universidad de Córdoba.

(1989): "Paleolítico en la provincia de Córdoba. La Barqueta (Palma del Río)", Ariadna, 6, p.104-118. Centro Municipal de Estudios Locales de Lora del Río.

ASQUERINO, M. D. (1988): "El Paleolítico en Córdoba. Estado actual de su conocimiento", Estudios de Prehistoria Cordobesa, 4, p.3-17.

BARROSO, C.; DURAN, J.J.; MOLINA, F. y MORGADO, A. (1989): "El glacís-terraza de Aljaima (Málaga) y su industria achelense", II Reunión del Cuaternario lbérico, Resúmenes, p.34-35, Madrid.

BEGUIRISTAIN, M.A. (Ined): "Materiales paleolíticos de la Colección Casas Morales, conservados en el Museo Arqueológico de Córdoba", VIII Simposium de Prehistoria Peninsular: Prehistoria y Protohistoria de la Cuenca del Guadalquivir (Córdoba, 1976).

BOTELLA, M. (1975): "El cazadero achelense de La Solana del Zamborino (Granada)", XIII C.N.A.: 175-184. (1976): "Excavaciones arqueológicas en el yacimiento achelense de La Solana de Zamborino, Fonelas (Granada), 1972-73", Not.Arq.His. Prehistoria 5: 25-31.

BOTELLA, M.;MARTINEZ, C. y CARDENAS, F.J. (1986): "Industria musteriense y achelense en Cueva Horá (Darro, Granada)", Homenaje a Luis Siret: 79-93. Sevilla.

BORDES, F.; VIGUIER, C. (1969): "Présence de galets taillés de type ancien dans la règion de Carmona (province de Sevilla, Espagne)", C.R. Acad Sc.París ,t. 269, Serie D, p.1946-1947.

BREUIL, H. (1914): "Stations chelléennes de la province de Cadíx", Insitut Française d'Anthropologie, Vol.II, p.67-79. París.

(1917): "Observations sur les Terres Noires de la Laguna de la Janda", L'Anthropologie, t.XXVIII, 236-240. París.

CABRE, J.; WERnERT, P. (1916): "El Paleolítico Inferior en Puente Mocho", Comisión de Investigaciones Paleontológicas y Prehistóricas, 11. Madrid.

CARBONELL, A. (1931): "Contribución al estudio de la Prehistoria Cordobesa: Indicios de una estación paleolítica en Santa Cruz", Boletín de la Academia de Ciencias, Bellas Letras y Nobles Artes de Córdoba, Año X, n.. 32 .

CARBONELL, E.; CANAL, J. (1981): "El tecno-complejo con cantos tallados de Rota I (Cádiz)", Vª Reunión del Grupo Español de Trabajo del Cuaternario. Actas y Guías de Excursiones, p.162-75. Universidad de Sevilla.

CARRASCO, J.; ANIBAL, C.; TORO,I.; ALMOHALLA, M.; GAMIR, J. (1979): "El yacimiento paleolítico de Puente Mocho. (Beas de Segura, Jaén). Nuevos hallazgos", Boletín del Instituto de Estudios Giennenses, n.o XCIX, Jaén, p.1-17.

CARRASCO, J. y otros. (1986): El poblamiento antiguo en la tierra de Loja. Ayuntamiento de Loja (Granada), p.256, XX lám..

CARRASCO, J.; TORO, I.; ALMOHALlA, M.; GAMIZ, J.; MALPESA, M. (1981): "Nuevos yacimientos paleolíticos en la provinica de Jaén", Grupo de Estudios Prehistóricos, Memoria de Actividades n.o I. La Carolina (Jaén), p.11-19, 13 lám..

CASAS, A. (1964): "El Paleolítico Inferior en la Campiña de Córdoba", Boletín de la Real Academia de Córdoba de Ciencias, Bellas Letras y Nobles Artes, n.․․ 86.

CASTIÑEIRA, J.; ALVAREZ, G.; GARCIA, J.M.; GOMEZ, F.; MARTIN, J.; RODRIGUEZ, J. (1989): "Evidencias paleolíticas en las terrazas de los ríos Tinto y Odiel (Huelva)", El Cuaternario en Andalucía Occidental, Aequa. Monografías, 1, 59-66.

CASTIÑEIRA, J.; GARCIA, J.M. (1985): "Paleolítico Inferior y Medio en la provinica de Huelva". Actas de la I Reunión del Cuaternario Ibérico, vol.II, p.61-78. Lisboa.

(1986): "Paleolítico y Epipaleolítico en Huelva", Huelva y su provincia, vol.II, cap.I. Ed.Tartessos. Sevilla. 
CASTIÑEIRA, J.; GARCIA, J.M.; ALVAREZ, G.; MARTIN, J. (1988): "Estado actual de las investigaciones paleolíticas de la provincia de Huelva", Trabajos de Paleolítico y Cuaternario, p.7-25. Sevilla.

DIAZ DEL OLMO, F.; VALLESPI, E. (1988): "Secuencia fluvial y paleolítica del Guadalquivir: terrazas y conexiones arqueológicas (Sevilla)", Trabajos de Paleolítico y Cuaternario, p.53-57. Sevilla.

DIAZ DEL OLMO, F.; VALLESPI, E.; ALVAREZ, G. (1985): "Formaciones cuaternarias y conexiones paleolíticas del Bajo Guadalquivir, provinica de Sevilla", Anuario Arqueológico de Andalucía, 1985, II, 13-16. (1986): "Formations superficielles détritiques et séquence paléolithique du Bas Guadalquivir (SW de l'Espagne)", Mediterranée, n.․ 3, p.61-65.

(1986): "Formaciones cuaternarias y conexiones paleolíticas del Bajo Guadalquivir, provinica de Sevilla", Anuario Arqueológico de Andalucía, 1986, II, p.7-11.

(1997): "Formaciones cuaternarias y conexiones paleolíticas del Bajo Guadalquivir, (Sevilla. Sector La Campana-Carmona), Anuario Arqueológico de Andalucía, 1987, II, 29-32.

DIAZ DEL OLMO, F.; VALLESPI, E. y Otros (1988): "Evolución geomorfológica, secuencia paleolítica y geomorfología reciente del sistema fluvial del Bajo Guadalquivir", Aluvionamientos cuaternarios de la Depresión Inferior del Guadalquivir. AEQUA, Grupo Andaluz de Cuaternario, p.37-66.

DIAZ DEL OLMO, F.; VALLESPI, E.; BAENA, R.; RECIO,J.M. (1989): "Terrazas pleistocenas del Guadalquivir occidental: geomorfología, suelos, paleosuelos y secuencia cultural", El Cuaternario en Andalucía Occidental, AEQUA, monografías, 1, p.33-42.

FERNANDEZ-CHICARRO, C.; MATEOS, V.; RAMIREZ DELGADO, J.R. (1956): "Materiales del paleolítico inferior hallados en las proximidades de la capital hispalense, que se conservan en el Museo Arqueológico de Sevilla", Congresos Internacionales de Ciencias Prehistóricas y Preotohistóricas. Actas de la IV sesión, 273275, lám..

FERNANDEZ-LLEBREZ, C. (1988): "Los yacimientos paleolíticos de la Depresión de La Janda (Provincia de Cádiz), Congreso Internacional El Estrecho de Gibraltar, Ceuta, 1987. Actas, Tomo I. UNED. Madrid.

GARCIA RINCON, J.M. (1987): "Excavación de urgencia en la Glorieta (Punta Umbría, Huelva), 1985", Anuario Arqueológico de Andalucía, 1985, III, 171-173.

(1987): "Prospección arqueológica del Paleolítico y Epipaleolítico de la provinica de Huelva", Anuario Arqueológico de Andalucía, 1986, II, 23-53.

GARCIA RINCON, J.M.; CASTIÑEIRA, J.; ALVAREZ, G.; MARTIN, J.; GOMEZ, F. (1990): "Resultado preliminar de la II Campaña de prospección superficial de la Tierra Llana de Huelva", Anuario Arqueológico de Andalucía, 1987, p.22-29.

GIBERT, J.; AGUSTI, J.; MOYA-SOLA, S. (1983): "Presencia de Homo sp. en el nacimiento del Pleistoceno Inferior de Venta Micena (Orce, Granada)", Paleontologia i Evolució. Publicació Especial, Institut de Paleontología, Sabadell.

GIBERT, J. (1986): "El nacimiento de Venta Micena (Orce, Granada). Su importancia, sección antropogámica y características paleoantropológicas del fragmento de cráneo de Homo sp. Homenaje a Luis Siret, p.37-48.

GIBERT,J.; MARTINEZ,B.; JIMENEZ,C.; FERNANDEZ,C.; IGLESIAS,A.; ARRIBAS,A.; RIBOT,F. y VARTUCA,f. (1989): "Estudio preliminar de las industrias líticas del Pleistoceno Inferior de las Cañadas de Vélez y el Salar (Orce,Granada)", Los restos humanos de Orce y Cueva Victoria, p.363-378. Institut Paleontologic. Gr.m.Consafont.

GILES, F. (1978): "Prehistoria de la Laguna de la Janda: nuevas aportaciones", Boletín del Museo de Cádiz, I, Cádiz, p. 7-17 y lám.

GILES, F.; SANTIAGO, A. (1988): "Avance al estudio del Paleolítico Inferior en la laguna de Medina, curso inferior del río Guadalete (Jerez de la Frontera, Cádiz)", Trabajos de Paleolítico y Cuaternario. Universidad de Sevilla, p.131-149.

GILES, F.; SANTIAGO, A.; GUTIERREZ, J.M.; MATA, E.; AGUILERA, L. (1989): "El poblamiento paleolítico en el valle del río Guadalete (Cádiz)", El Cuaternario en Andalucía Occidental. AEQUA monografías, 1, 43-57.

HERNANDEZ PACHECO, E. (1915): "Las tierras negras del extremo Sur de España y sus yacimientos paleolíticos", Trabajos del Museo Nacional de Ciencias Naturales. Serie Geología, n.o 13. 
LEIVA, J.A. (1977): "El Paleolítico de la provinica de Málaga", XIV Congreso Nacional de Arqueología, Vitoria, 1975, p.113-120, lám..

LOPEZ, M.; SORIA, M. (1976): "El yacimiento de cuarcitas talladas de La Calera", Zephyrus, XXVI-XXVII. Universidad de Salamanca, p.111-127.

OBERMAIER, H. (1916): "El Hombre Fósil”, Comisión de Investigaciones Paleontológicas y Prehistóricas, memoria n. 9 9. Madrid.

(1925): "El Hombre Fósil", Comisión de Investigaciones Paleontológicas y Prehistóricas, memoria n.. 9. Segunda Edición refundida y simplificada. Madrid.

PEREDA, C.; MARTINEZ, F. (1988): "Dos nuevas localizaciones paleolíticas en superficie de cantos tallados en los alrededores de Sevilla", Trabajos de Paleolítico y Cuaternario, p.87-101. Sevilla.

QUEROL, M.”.A.; SANTONJA, M. (1983): "El yacimiento de cantos trabajados de El Aculadero (Puerto de Sta. M.". ., Cádiz)", Excavaciones Arqueológicas en España, 130, 270 p..

RAMIREZ, J.R.; FERNANDEZ-LLEBREZ, E.; MATEOS, V. (1989): “Aproximación al estudio del Cuaternario de la Laguna de la Janda (Cádiz)", El Cuaternario en Andalucía Occidental, AEQUA monografías, 1, 105111 .

RAMOS, J. (1988): El poblamiento prehistórico del Alto Vélez hasta la Edad del Bronce. Biblioteca Popular Malagueña, p.34-38.

RODRIGUEZ, I. (1988): "Evidencias paleolíticas en el río Corbones", Trabajos de Paleolítico y Cuaternario. Universidad de Sevilla, p.103-115.

RODRIGUEZ, J.; MAYORAL, E.; CASTIÑEIRA, J.; GARCIA, J.M. (1988): "Evolución geomorfológica del litoral Suratlántico Ibérico", Aluvionamientos cuaternarios en la Depresión Inferior del Guadalquivir, AEQUA, Grupo Andaluz del Guadalquivir, p.35.

RUIZ BUSTOS, A. (1975): Estudio sistemático y ecológico sobre la fauna del Pleistoceno Medio en las Depresiones granadinas. El yacimiento de Cúllar de Baza I. Tesis doctoral.Universidad de Granada, Fac. de Ciencias, Zoología, 239 p., 60 lám..

(1984): "El yacimiento paleontológico de Cúllar de Baza I", Investigación y Ciencia, n.. 91, p. 20-28.

RUIZ BUSTOS, A.; MICHAUX, I. (1976): "Le site préhistorique nouveau de Cúllar de Baza I (province de Granade, Espagne) d'âge pléistocène moyen. Etude préliminaire et analyse de la faune des rougeurs", Gèologie Méditerranéenne, t.III, n. $\mathrm{o}$ 3, Universite de Provence, p. 173-182.

SAEZ, B. (1956): "Arqueolítico y Paleolítico: Carmona", Noticiario Arqueológico Hispánico, III y IV. Cuadernos 1-3, 1954-1955. Madrid.

SANCHEZ, C. (1975): "Nuevos yacimientos paleolíticos en Jaén", XIII Congreso Nacional de Arqueología, (Huelva, 1973), p. 173 y 174, lám.. Zaragoza.

SANCHEZ, C.; GARCIA, F. (1977): "Paleolítico en Cortijos «Piedras de Sta.Inés» y «Angulo», Villanueva de la Reina, Jaén", Xiv Congreso Nacional de Arqueología, Vitoria, 1975, p. 121-122 y lám..Zaragoza.

SANTONJA, M.; QUEROL, M.A. (1988): "Paleolítico Inferior arcaico en la Depresión del Guadalquivir", VIII Symposium de Prehistoria Peninsular: Prehistoria y Protohistoria de la Cuenca del Guadalquivir, Córdoba, 1976. Trabajos de Paleolítico y Cuaternario, Universidad de Sevilla, p.39-51.

SANTONJA, M.; VILA, P. (1990): "The Lower Paleolithic of Spain and Portugal", Journal of World Prehistory, vol 4, n. ${ }^{\circ} 1,45-94$.

SANTIAGO, A.; GILES, F. (1988): "Paleolítico Inferior en la Laguna de Medina, Investigaciones en el curso bajo del río Guadalete", Revista de Arqueología, Año IX, n.․ 87, p.7-14.

SOLANES, M." .C.; GARCIA, M. (1983): "Carta Arqueológica del término municipal de Marbella", Cilniana, n.. 6. Ayuntamiento de Marbella, 74 p..

TERRA, H. de (1956): "Climatic terraces and the Paleolihique of Spain", Libro homenaje al Conde de la Vega de Sella. Oviedo.

TORO, I.; RAMOS, M. (1988): "Nueva estación paleolítica al aire libre en la cuenca media del río Genil. El yacimiento achelense del Cortijo del Calvillo de Fuente Camacho (Loja, Granada)", Trabajos de Paleolítico y Cuaternario, p.151-163. Universidad de Sevilla.

VALLESPI, E. (1983): “El poblamiento paleolítico inicial”, Sevilla y su provincia, t.II, p.15-21. Sevilla. 
(1986): "El Paleolítico Inferior y Medio en Andalucía", Homenaje a Luis Siret (1934-1984), Consejería de Cultura de La Junta de Andalucía, p.59-66.

(1987): "Un bifaz achelense del tramo Subbético Exterior Occidental", Anuario Arqueológico Andaluz, 1985, Junta de Andalucía, p.288-290.

(1988): "Venta Micena, de Orce, cinco años despues. Anotación a sus anunciadas industrias líticas", Raña, Boletín Informativo de la Asociación Española para el estudio del Cuaternario, n.․ 5, págs. centrales I-VI.

(1988): "Paleolítico Medio de aspecto postachelense en la Depresión Inferior del Guadalquivir", Homenaje al Prof. E.Ripoll Rerelló, Espacio, Teimpo y Forma, serie I, Prehistoria, t.I, p.85-91, UNED.

(1989): "Paleolítico Medio indeterminado de aspecto postachelense, en ámbitos fluviales del Sur y Centro de la Península Ibérica", Veleia, p.6,7-20.

VALLESPI, E.; ALVAREZ,G.; PEREZ SINDREU, F.; RUFETE,P. (1986): "Nuevas aportaciones onubenses al Paleolítico Inferior y Medio", Huelva en su Historia, 1, p.43-56, Colegio Universitario de la Rábida.

VALLESPI, E.; ESCACENA,J .L.; RAMOS,J . (e.p.): "Un bifaz de La Barrosa, del Achelense Superior o su tradición inmediata, del frente atlántico andaluz”. I Congreso de Estudios Locales de la Provincia de Cádiz.

VALLESPI, E. y AMORES, F. (1988): "Dos bifaces del Bajo Guadalquivir Sevillano", Trabajos de Paleolítico y Cuaternario, p.117-129. Sevilla.

VALLESPI, E.; ALVAREZ, G.; AMORÉS, F.; ESCACENA,J .L. (1982): "Complejos de cantos tallados y bifaces en el Bajo Guadalquivir: perspectivas de su estudio", Grupo Español de Trabajo del Cuaternario, $5^{\mathrm{a}}$ Reunión, Conferencias p. 79-94. Universidad de Sevilla.

VALLESPI, E.; AMO, M. del; ALVAREZ, G. (1981): "Primeras evidencias paleolíticas de la provincia de Huelva", Huelva Arqueológica, p.9-26.

VALLESPI, E.; CRUZ-AUÑON, R.; RIVERO, E. (e.p.): "Bifaz achelense del Bajo Genil, en Santaella, Córdoba", Cuadernos de Prehistoria de la Universidad de Granada.

VALLESPI, E.; DIAZ del OLMO, F.; ALVAREZ, G.; VALLESPI, E. (1988): "Industrias y secuencia del Paleolítico Inferior y Medio en el Bajo Guadalquivir, provinica de Sevilla", Trabajos de Paleolítico y Cuaternario, p.59-85, Sevilla.

(1988): "Secuencia paleolítica del Bajo Guadalquivir", Revista de Arqueología, n.. 82, p.8-17.

VALLESPI, E.; RAMOS, J.; MARTIN, E. (1989-90): "Bifaz del Alto Vélez, del Achelense Superior o de su tradición inmediata", Mainake, XI-XII, p.7-10.

VEGA, TOSCANO L.G. (1984): "Crisis de la credibilidad en Arqueología", Revista de Arqueología, n.. 39, p.56-58. Madrid.

(1989): "Ocupaciones humanas en el Pleistoceno de la depresión de Guadix-Baza: elementos de discusión", Geología y Paleontología de la Cuenca de Guadix-Baza, p.327-345. Madrid.

VIGUIER, C.; THIBAULT, C. (1973): "Nouveaux élèments de datation des formations de Pièdemont de la Sierra Morena à l'Ouest de Séville", Estudios Geológicos, vol.XXIX, p.351-354. Madrid.

VIGUIER, C. (1974): Le Neogene de l'Andalusie Nord Occidentale (Espagne). Histoire Géologique du Bassin du Bas Guadalquivir. Thèse d'Etat. Burdeos.

ZAZO, C.; GOY, J.L.; DABRIO, C.J.; CIVIS, J.; BAENA,J . (1985): "Paleogeografía de la desembocadura del Guadalquivir al comienzo del Cuaternario. (Provinica de Cádiz, España), Actas de la I Reunión del Cuaternario Ibérico, 1, p.461-472. Lisboa. 